\section{Pitch discrimination is better for synthetic timbre than natural musical instrument} timbres, despite familiarity

\author{
Emma Holmes \\ Department of Speech Hearing and Phonetic Sciences, University College London, London, \\ WC1N 1PF, U.K. \\ Brain and Mind Institute, University of Western Ontario, London, Ontario, N6A 5B7, Canada \\ emma.holmes@ucl.ac.uk \\ Elizabeth Kinghorn \\ Don Wright Faculty of Music, University of Western Ontario, London, Ontario, N6A 3K7, \\ Canada \\ elizabethkinghorn@gmail.com
}

4

5

\section{Lucy M. McGarry}

Brain and Mind Institute, University of Western Ontario, London, Ontario, N6A 5B7, Canada lmcgarry@uwo.ca

\section{Elizabeth Busari}

UCL Ear Institute, University College London, London, WC1E 6BT, U.K.

ebusari@live.co.uk 
24 Biosciences Institute, Faculty of Medical Sciences, Newcastle University, Newcastle upon Tyne NE2 4HH, U.K.

26 Wellcome Centre for Human Neuroimaging, UCL Queen Square Institute of Neurology, University College London, London, WC1N 3AR, U.K. tim.griffiths@newcastle.ac.uk

31 Brain and Mind Institute, University of Western Ontario, London, Ontario, N6A 5B7, Canada;

32 School of Communication Sciences and Disorders, University of Western Ontario, London,

ijohnsru@uwo.ca

36 Running title: Pitch thresholds depend on timbre

37 Date uploaded: 24th September 2021 
ABSTRACT

Pitch discrimination is better for complex tones than for pure tones, but how more subtle

40 differences in timbre affect pitch discrimination is not fully understood. This study compared

41 pitch discrimination thresholds of flat-spectrum harmonic complex tones with those of natural

42 sounds played by musical instruments of three different timbres (violin, trumpet, and flute). To

43 investigate whether natural familiarity with sounds of particular timbres affects pitch

44 discrimination thresholds, this study recruited musicians who were trained on one of the three

45 instruments. We found that flautists and trumpeters could discriminate smaller differences in

46 pitch for artificial flat-spectrum tones, despite their unfamiliar timbre, than for sounds played by

47 musical instruments, which are regularly heard in everyday life (particularly by musicians who

48 play those instruments). Furthermore, thresholds were no better for the instrument a musician

49 was trained to play than for other instruments, suggesting that even extensive experience

50 listening to and producing sounds of particular timbres does not reliably improve pitch

51 discrimination thresholds for those timbres. The results show that timbre familiarity provides

52 minimal improvements to auditory acuity, and physical acoustics (i.e., the presence of equal-

53 amplitude harmonics) determine pitch-discrimination thresholds more than does experience with

54 natural sounds and timbre-specific training.

\section{KEYWORDS}

57 Music; training; pitch; discrimination 


\section{INTRODUCTION}

The ability to discriminate small pitch differences may be particularly beneficial for

61 musicians. For many orchestral instruments, a musician's ability to detect small differences in

62 frequency is critical for playing the correct note and also, in some instances (e.g., violin), for

63 tuning the instrument. The threshold, or 'just noticeable difference' (JND), for discriminating

64 pitch is often measured by presenting two sounds sequentially and asking listeners whether the

65 two sounds have the same or a different pitch, or which sound is higher or lower in pitch.

The JND depends on acoustic properties of the sounds to be discriminated: Listeners can

67 detect smaller differences in pitch between two harmonic complex tones than between two pure

68 tones (Flanagan and Saslow, 1958; Micheyl et al., 2006; Zeitlin, 1964) demonstrating that

69 listeners utilize the harmonics of complex tones to improve pitch judgements. Better

70 discrimination at particular frequencies cannot account for this finding, because the JND for a

71 complex tone is better than the best pure-tone JNDs of its component frequencies (Faulkner,

72 1985). At the same time, pitch discrimination depends on the combination of harmonics that are

73 present in a complex tone (Houtsma \& Smurzynski, 1990; Miyazono \& Moore, 2013; Moore,

74 Glasberg, \& Proctor, 1992). Houtsma and Smurzynski (1990) presented complex tones with a

75 missing fundamental of $200-300 \mathrm{~Hz}$, containing a subset of harmonics (those above 7-25 in the

76 harmonic series). JNDs were worse for complex tones that only contained frequencies above the

$7713^{\text {th }}$ harmonic than for those that also contained lower frequencies (7-12 in the harmonic series).

78 Thus, the timbre of a harmonic complex tone influences the smallest pitch differences that a

79 listener can perceive.

80 Sounds in the natural world are not complex tones with a flat spectrum or with their

81 harmonics selectively removed, like those used in the aforementioned experiments. Rather, the 
82 resonant properties of the production system (e.g., human vocal tract or body of a musical

83 instrument) produce sounds with formants at the positions of resonances and, more generally,

84 different intensities at different frequencies. On one hand, natural sounds contain less acoustic

85 information than flat-spectrum complex tones, leading to the hypothesis that pitch discrimination

86 should be worse for natural sounds. On the other hand, the ability to discriminate differences in

87 pitch can be improved through training (Amitay et al., 2005; Ari-Even Roth et al., 2003; Atienza

88 et al., 2002; Delhommeau et al., 2002; Demany, 1985; Demany and Semal, 2002; Grimault et al.,

89 2002; Irvine et al., 2000; Menning et al., 2000; Micheyl et al., 2006; Sinnott et al., 1985),

90 demonstrating that discrimination thresholds are not fixed, but are instead influenced by prior

91 experience. Possibly, prior experience with sounds in the natural world might lead to better pitch

92 discrimination for tones with natural (familiar) than unnatural (unfamiliar) spectra; this effect

93 may be large enough to override the benefit obtained from the greater acoustic information

94 available in unnatural, flat-spectrum harmonic-complex tones.

95 Relatively few studies have measured pitch discrimination of sounds with natural spectra.

96 Two studies measured pitch discrimination of artificial vowels (Flanagan and Saslow, 1958) and

97 of a female speaking the vowel "ah" (Moore, Estis, Gordon-Hickey, \& Watts, 2008). Flanagan

98 and Saslow (1958) found better discrimination of artificial vowels than of pure tones, showing

99 that the advantage for discriminating complex, compared to pure, tones also applies to sounds

100 with more natural spectra. To our knowledge, Moore et al. (2008) was the only group to compare

101 natural (vocal) and artificial complex tones. They found no difference in pitch discrimination

102 between the natural female voice and the artificial complex tone, although the vowel that was

103 spoken by the female talker was missing word or sentence context that we naturally encounter,

104 which may help us to discriminate pitch when listening to speech in natural settings. Other 
105 studies using natural (Allen and Oxenham, 2014; Pitt, 1994, Experiment 1; Vurma et al., 2011)

106 and artificial (Krumhansl and Iverson, 1992; Singh and Hirsh, 1992; Warrier and Zatorre, 2002)

107 timbres have demonstrated that a difference in timbre between a pair of tones influences

108 judgements of pitch in variations of same-different paradigms. However, these papers do not

109 report comparisons between timbres when the timbre of the two tones was the same within each

110 trial. In a task in which participants classified pitch as high or low, Pitt (1994, Experiment 2)

111 found no difference depending on whether the stimuli were trumpet or piano tones; however, the

112 pitch difference (294 or $417 \mathrm{~Hz}$ ) was large and accuracy was > 90\% correct, so this null result

113 could be explained by a ceiling effect.

114 Other studies have examined effects of timbre in pitch interval discrimination tasks,

115 although the findings are mixed. Zarate, Ritson, and Poeppel (2013) compared pitch interval

116 discrimination of pure tones, piano tones, flute tones, and synthetic voices. However, contrary to

117 the aforementioned pitch discrimination tasks, they found that pitch interval discrimination was

118 most accurate for pure tones. Russo and Thompson (2005) found that judgements of pitch

119 interval size were affected by changes in artificial timbre between the first and second tone of the

120 interval, but when the timbre was constant, pitch interval size judgements did not differ between

121 "bright" and "dull” timbres.

122 The notes played by musical instruments vary naturally in timbre, and musicians have 123 extensive experience with the musical instrument on which they are trained. Musical training 124 might be considered a special instance, and more naturalistic form, of pitch discrimination 125 training. Indeed, musicians perform substantially better (by a magnitude of 2-4) on pitch 126 discrimination tasks than non-musicians, both when they are required to discriminate two tones 127 that are presented sequentially (Besson et al., 2007; Brown et al., 2017; Kishon-Rabin et al., 
128 2001; Magne et al., 2006; Micheyl et al., 2006) and when they are required to discriminate tones

129 in 10-tone sequences (Spiegel and Watson, 1984). Although musicians have not been found to 130 discriminate timbre better than non-musicians (Allen and Oxenham, 2014), Micheyl et al. (2006)

131 report a larger musician advantage for discriminating the pitches of complex compared to pure

132 tones, consistent with the idea that musicians' natural experience with complex tones improves

133 pitch discrimination most for complex tones and less for sounds that differ vastly in timbre from

134 trained sounds (i.e., pure tones). Musicians might be expected to show better pitch discrimination

135 for sounds of an instrument they have been trained to play, given that they have extensive

136 experience making pitch judgements for the timbre of that instrument compared to other sounds

137 they cannot produce. Consistent with this idea, Miyazaki (1989) found that piano players with

138 absolute pitch were best at identifying the pitch of natural piano tones (91.6\% correct),

139 intermediate at identifying the pitch of artificial piano sounds (74.4\%), and worst at identifying

140 the pitch of pure tones (80.4\%); although, these results cannot distinguish whether this advantage

141 reflects training on the piano specifically, or a more general advantage for natural compared to

142 artificial sounds. Also, effects of timbre on pitch identification in individuals with absolute pitch

143 may differ from more general effects of timbre on pitch discrimination.

144 Several mechanisms could underlie better pitch discrimination of sounds played on

145 instruments that musicians have been trained to play. Experience could improve pitch

146 discrimination by enabling better predictions of where in the frequency spectrum or when in time

147 (based on knowledge of the temporal envelope) to listen. Alternatively, musicians would be

148 expected to have strong sensorimotor mappings for pitches of instruments that they play; these

149 would be weaker or nonexistent for other instruments. Such mappings, which may provide

150 another way to represent pitch information, might improve pitch discrimination. For instruments 
151 capable of fine changes in pitch (e.g., violin, flute, trumpet), these mappings may be even more

152 fine-tuned for pitch; to play the correct pitch, musicians must make rapid motor (mouth or

153 finger) micro-adjustments and be able to hear how these affect the pitch of the sound they

154 produce. Indeed, Hafke-Dys, Preis, and Trojan (2016) demonstrated that violinists are capable of

155 compensating for perturbations in pitch while playing a series of notes, even when the pitch

156 perturbations were lower than their perceptual thresholds measured when they heard but did not

157 play the same notes. Musicians show enhanced neural responses to notes played on instruments

158 that they play, compared to notes played on instruments they have never been trained to play

159 (Pantev et al., 2001; Shahin et al., 2008; Strait et al., 2012), which provides a neural substrate by

160 which perception could be improved for trained instruments. Nevertheless, whether pitch

161 discrimination is better for trained than untrained instruments is currently unknown.

162 Here, we compared musicians' and non-musicians' pitch-discrimination thresholds for

163 musical tones (played on the violin, trumpet, and flute) and synthetic flat-spectrum harmonic

164 complex tones. We presented participants with two bars of a 4-tone sequence that contained a

165 deviant-pitch tone in the second bar. We showed participants musical notation for the first bar

166 before the sequence began, so that they knew in advance the correct pitches of the tones.

167 Consistent with previous findings (e.g., Besson et al., 2007; Brown et al., 2017; Kishon-Rabin et

168 al., 2001; Magne et al., 2006; Micheyl et al., 2006; Spiegel and Watson, 1984), we expected to

169 find better pitch discrimination in musicians than non-musicians. Crucially, we recruited three

170 groups of musicians who were trained to play one of the instruments (violin, trumpet, or flute)

171 that they heard in the experiment, enabling us to compare differences in thresholds due to

172 acoustics and familiarity. If thresholds are primarily determined by acoustic content, pitch-

173 discrimination thresholds should be better for flat-spectrum harmonic complex tones than for 
174 musical tones. Whereas, if the effect of experience is large enough to compensate for the

175 additional acoustic information in flat-spectrum harmonic-complex tones, thresholds for musical

176 tones should be as good or better than thresholds for complex tones — and be best for the musical

177 instrument that the listener has learnt to play.

178 II. METHODS

179 A. Participants

180 We excluded data from 4 participants: three did not meet the criteria for normal hearing

181 (average pure-tone thresholds averages greater than $20 \mathrm{~dB}$ HL at octave frequencies between 0.5 182 and $4 \mathrm{kHz}$ ) and one did not respond on $15 \%$ of trials. We analysed the data from 51 participants

183 (16 male, 35 female) aged $18-58$ years $($ median $=24.0$ years, interquartile range $[\mathrm{IQR}]=10.5)$

184 with normal hearing (average pure-tone thresholds at octave frequencies between 0.5 and $4 \mathrm{kHz}$ 185 of $15 \mathrm{~dB}$ HL or better in each ear).

186 Non-musicians $(\mathrm{N}=14)$ were required to have no more than two years of musical 187 training: our non-musicians had either never been trained on an instrument $(\mathrm{N}=9)$, or had learnt 188 an instrument (the piano, recorder, or bongo drums) for less than or equal to 2 years between the 189 ages of 8 and 15 years. Musicians had been trained to play either the violin $(\mathrm{N}=15)$, flute $(\mathrm{N}=$ 190 16), or trumpet $(\mathrm{N}=6)$, but had never been trained to play either of the other two instruments. 191 Musicians had 3-53 years (median = 15 years, IQR $=7$ ) of musical training, which they started 192 age 3-16 years (median = 7 years, IQR = 3). They had started playing the violin, flute, or 193 trumpet at age 3-34 years (median = 8 years, IQR = 3) and had played the instrument for 2-53 194 years $($ median $=11$ years, IQR $=12)$. Table 1 displays the demographics of musicians separated 195 by training group (violinists, flautists, and trumpeters); independent-samples Kruskal-Wallis 
196 tests showed no significant differences in these demographics among training groups $[H(37) \leq$ $197 \quad 5.5, p \geq 0.063]$

198

199 Table 1. Demographics for the three musician groups. Table displays medians (except in the final 200 row, where percentages are displayed), with interquartile ranges in brackets.

\begin{tabular}{llll}
\hline & Violinists & Flautists & Trumpeters \\
\hline Age & $27.0(12.8)$ & $21.9(5.8)$ & $24.9(6.4)$ \\
Years of music training & $19.0(7.5)$ & $14.5(4.3)$ & $13.5(9.3)$ \\
Age started music training & $6.0(4.0)$ & $6.5(2.3)$ & $7.5(3.3)$ \\
Years playing trained instrument & $19.0(10.0)$ & $9.0(9.0)$ & $11.0(7.3)$ \\
Age started playing trained instrument & $7.0(2.5)$ & $10.0(3.3)$ & $10.0(2.3)$ \\
Percentage currently practicing trained instrument $(\%)$ & 73.3 & 43.8 & 66.7
\end{tabular}

204 Board and the UCL Research Ethics Committee. Informed consent was obtained from all 205 participants. 17 participants (6 violinists, 7 flautists, and 4 trumpeters) took part at Western 206 University and 34 (9 violinists, 9 flautists, 2 trumpeters, and 14 non-musicians) participated at 207 University College London (UCL).

\section{B. Apparatus}


Acoustic stimuli were presented through an external sound card (Steinberg UR22 sound

212 card [Steinberg Media Technologies] at Western University or ESI Maya 22 USB [ESI

213 Audiotechnik $\mathrm{GmbH}$ ] at UCL). Stimuli were delivered binaurally through earphones (Etymotic

214 Research ER1 earphones [Etymotic Research, Inc.] at Western University), which were sealed in

215 the ear canal of the participant with disposable foam inserts, or circumaural headphones

216 (Sennheiser HD 380 Pro [Sennheiser electronic GmbH \& Co. KG] at UCL).

\section{C. Stimuli}

We constructed 33 unique four-tone sequences using concert pitches between C4 (261.6

$219 \mathrm{~Hz})$ and C5 $(523.3 \mathrm{~Hz})$. The sequences included a range of patterns (tonal and triadic sequences,

220 chromatic sequences, and atonal patterns) and a variety of intervals and melodic contours.

221 Within each four-tone sequence, the musical pitch of every tone was different. Across sequences, 222 there was approximately equal representation of each of the 13 pitches in the C4-C5 octave.

223 Violin, trumpet, and flute tones were obtained from the Philharmonia Orchestra sound 224 sample database (http://www.philharmonia.co.uk; for examples, see Figure 1). We selected tones 225 of approximately 350-ms duration from the samples. We also created artificial flat-spectrum 226 harmonic complex tones of 350-ms duration using custom-written MATLAB scripts (version 227 2014b; The MathWorks, Inc.). These harmonic complex tones were created by summing sine 228 waves (with $0^{\circ}$ phase) at integer multiples of the fundamental frequency up to $20,000 \mathrm{~Hz}$. A 229 ramp time of $10 \mathrm{~ms}$ was applied to the beginning and the end of each tone. All stimuli were 230 normalised to the same root-mean-square (RMS) amplitude and were presented at a comfortable 231 listening level. 


\section{(a) Flute}
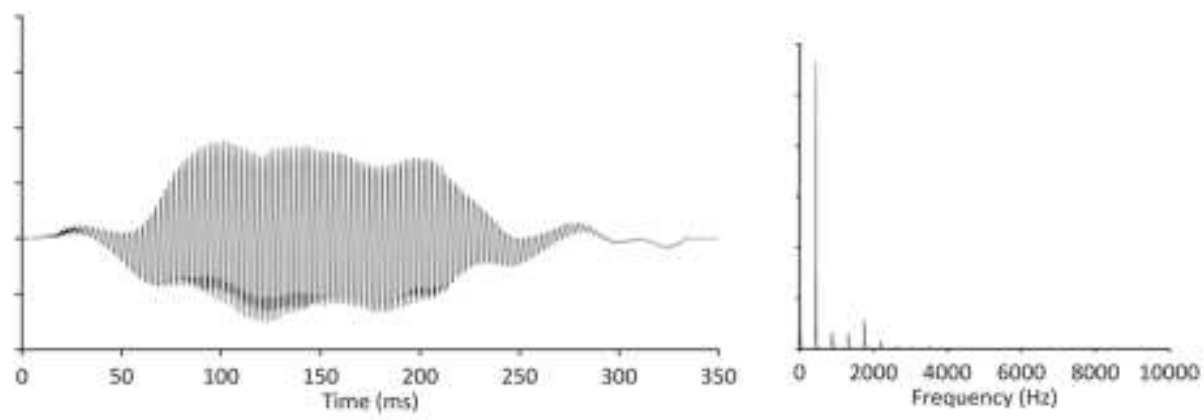

(b) Trumpet
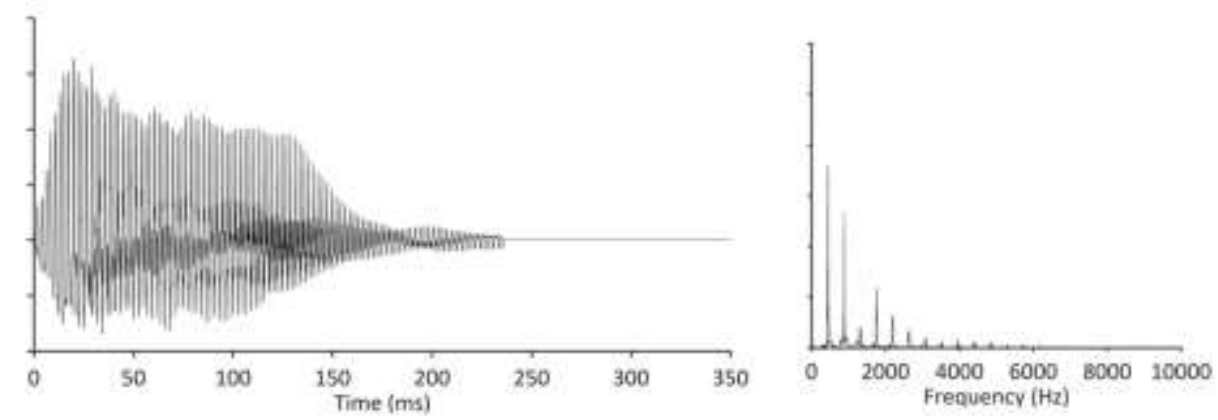

(c) Violin
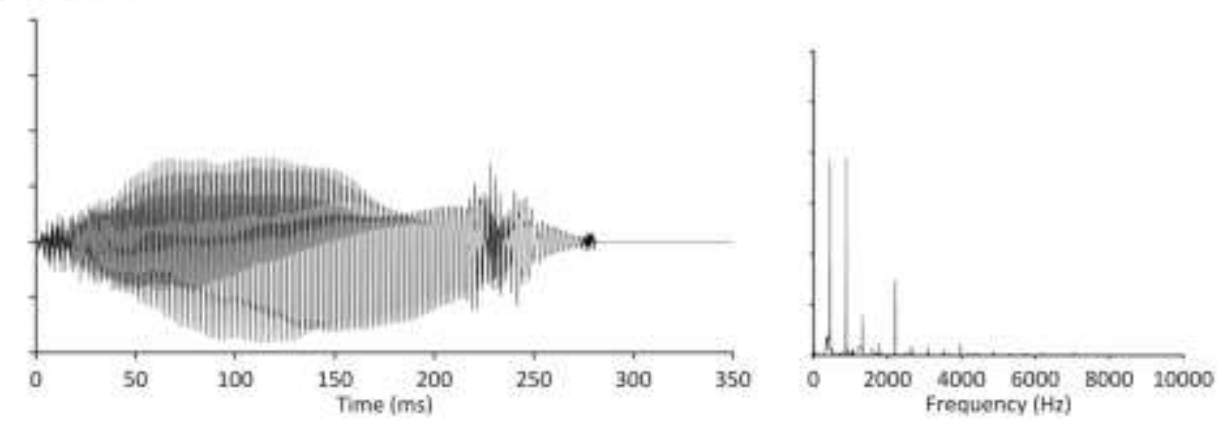

(d) Flat-spectrum complex tone


234 Figure 1. Waveforms (left) and spectra (right) of example stimuli at A4 pitch. (a) Flute, (b) 235 Trumpet, (c) Violin, (d) Flat-spectrum complex tone. 
237 To create deviant-pitch tones, we shifted the median pitch of individual tones upwards 238 (i.e., so that they sounded sharper than the original) using the 'Change Gender' function in Praat 239 (version 5.4.04; www.fon.hum.uva.nl/praat). This procedure changed the frequencies of the 240 harmonics within the tone at ratio intervals of 0.0005 , producing tones with higher pitches than

241 the originals. We chose to always shift the pitch upwards because thresholds for detecting pitch 242 increases and pitch decreases may differ (e.g., Salzberg, 1980).

243 We showed participants musical notation for the first bar of the sequence before the 244 sequence began, so that they knew in advance the correct pitches of the tones. Each of the 33 245 four-tone sequences was illustrated as one bar of music in common (4/4) time (Figure 2). Each 246 tone was displayed as a quarter note. The stimuli were written in simple Western notation using 247 Sibelius 8.0 (Avid Technology, Inc.). All sequences were written in the treble clef, but with no 248 time signature, key signature, dynamics, tempo, or other expressive markings indicated. Because 249 the acoustic stimuli did not distinguish between enharmonic equivalents (e.g., F-sharp and G-flat, 250 or B-natural and C-flat) the most common enharmonic equivalents were displayed with 251 approximately equal frequency across sequences. 


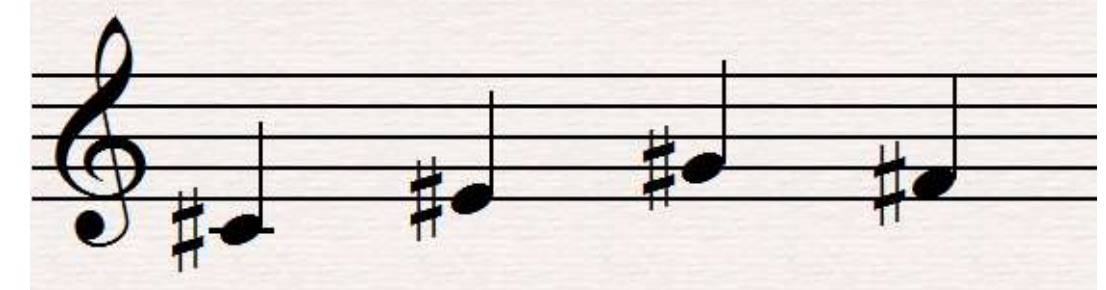

254 Figure 2. Musical notation for one of the 33 four-tone sequences, which was displayed to 255 participants before they heard the sequence.

\section{D. Procedure}

258 On each trial, the visual notation was presented on the screen for $1500 \mathrm{~ms}$. Next, after an 259 inter-stimulus interval of $700 \mathrm{~ms}$, participants heard two repetitions of the four tones that had 260 been displayed visually. Adjacent tones were presented with a stimulus onset asynchrony (SOA) 261 of $350 \mathrm{~ms}$. During the second presentation of each sequence, either the second or third tone of 262 the sequence was higher in pitch than the equivalent tone in the first presentation. Participants 263 had to respond which of the two tones was 'mistuned' in a two-alternative forced-choice 264 procedure. The tones for each trial were selected from either the set of violin tones, trumpet 265 tones, flute tones, or artificial harmonic complex tones.

266 We measured each participant's 70.0\% discrimination-threshold using a weighted up267 down adaptive procedure (Kaenbach, 1991; step size $=0.0005$, weighting ratio of 3:7). The 
268 starting value for each run was 0.0102 above the median pitch of the original tone (e.g., $264.3 \mathrm{~Hz}$

269 for an original pitch of $261.6 \mathrm{~Hz}$ ). The procedure stopped after 8 reversals. We adapted pitch for

270 each timbre (violin, flute, trumpet, artificial complex tone) in four separate, but interleaved, runs.

271 JNDs were calculated as the median of the last five reversals for each run. We express the $70.0 \%$

272 JND as the Weber fraction, which is calculated as the absolute difference in pitch at the $70.0 \%$

273 threshold divided by the reference pitch.

\section{E. Analyses} (https://doomlab.shinyapps.io/mote/).

To examine effects of Musicianship on pitch discrimination thresholds, we performed a 278 two-way mixed ANOVA, with Timbre (violin, flute, trumpet, and complex tone) as a within279 subjects factor and Musicianship (musician and non-musician) as a between-subjects factor. For 280 the musician group, we collapsed across flautists, violinists, and trumpeters.

To examine effects of instrument-specific training on pitch discrimination thresholds, we

282 analysed the data from musicians across the three Training groups (violinists, flautists, and 283 trumpeters). We analysed the results using a two-way mixed ANOVA, with Timbre as a within284 subjects factor and Training as a between-subjects factor. To examine differences between 285 Timbres - which were either natural musical instrument sounds or artificial flat-spectrum 286 harmonic complex tones-we performed (planned) paired-samples $t$-tests to compare thresholds 287 between the four Timbres.

288 Given that we expected to find differences in discrimination thresholds depending on 289 whether or not participants had learnt the instrument that produced the stimulus timbre, we 290 performed three planned independent samples $t$-tests: one compared thresholds for violin tones 
291 depending on whether or not participants had learnt to play the violin (i.e., violinists compared to 292 flautists and trumpeters), and the other two tests compared thresholds for flute and trumpet tones 293 depending on whether or not participants had learnt to play the flute or trumpet, respectively.

294 Given there was wide variability in musical history within each musician group, we 295 examined the relationship between these training factors and pitch discrimination thresholds. All 296 musicians $(\mathrm{N}=37)$ were included in these analyses. The training data violated the assumption of 297 normality, so we used Spearman's rank correlation coefficients to examine these relationships. 298 First, we calculated the difference in thresholds between the artificial flat-spectrum complex 299 tones and the average of the three natural musical instrument timbres. We then examined the 300 relationship between this threshold difference and (i) the number of years of musical experience 301 for each participant, and (ii) the age at which the participant began musical training. We also 302 calculated the threshold benefit for the trained instrument in each participant, defined as the 303 difference in thresholds between the trained instrument (e.g., violin tones for violinists) and the 304 average of the thresholds for the two other instruments (e.g., flute and trumpet tones for 305 violinists). We examined the relationship between the threshold benefit for the trained instrument 306 and (i) the number of years participants had played that instrument (e.g., the number of years 307 playing the violin for violinists) and (ii) the age at which participants had started learning that 308 instrument. In addition, we compared the threshold benefit for the trained instrument between 309 musicians who regularly played their trained instrument (who reported practicing every week, on 310 average; $\mathrm{N}=22)$ and those who were no longer practicing it $(\mathrm{N}=15)$, using an independent 311 samples $t$-test. 


\section{A. Musicians compared to non-musicians}

314 Figure 3a illustrates thresholds across the four timbres for musicians and non-musicians.

315 There was no significant main effect of Timbre $\left[F(3,147)=2.27, p=0.08, \omega_{p}{ }^{2}=0.02(95 \%\right.$ CI:

$3160.00-0.07)]$. However, there was a significant main effect of Musicianship $[F(1,49)=7.02, p=$ $3170.011, \omega_{p}^{2}=0.11$ (95\% CI: 0.00-0.30)]: Musicians (mean $=0.0147$, standard deviation [s.d.] $=$ 318 0.0044) had significantly smaller (i.e., better) thresholds than non-musicians (mean $=0.0183$, s.d. $319=0.0044)$.

320 There was also a significant interaction between Timbre and Musicianship $[F(3,147)=$ $3215.39, p=0.002, \omega_{p}^{2}=0.07$ (95\% CI: $\left.\left.0.00-0.15\right)\right]$. Independent-samples $t$-tests showed that 322 musicians had smaller thresholds than non-musicians for the flat-spectrum harmonic complex 323 tones $[t(15.97)=2.91, p=0.010, d=0.83(95 \%$ CI: $0.27-1.55)]$ and flute tones $[t(49)=3.23, p=$ $3240.002, d=0.92(95 \%$ CI: $0.36-1.66)]$, but not for the violin tones $[t(16.72)=0.50, p=0.62, d=$ 3250.14 (95\% CI: $-0.46-0.77)]$ or trumpet tones $[t(49)=0.67, p=0.50, d=0.19$ (95\% CI: $-0.41-$ 326 0.83)]. Looking at the interaction the other way using one-way repeated measures ANOVAs for 327 each group, there was a significant effect of Timbre for musicians $[F(3,108)=7.80, p<0.001$, $328 \omega^{2}=0.36(95 \%$ CI: $\left.0.20-0.48)\right]$ but not for non-musicians $\left[F(1.87,24.4)=1.72, p=0.20, \omega^{2}=\right.$ 3290.09 (95\% CI: 0.00-0.33)]. The significant effect of Timbre in musicians was underpinned by 330 smaller thresholds for flat-spectrum harmonic complex tones than for the three musical 331 instrument timbres [violin: $t(36)=3.88, p<0.001, d=0.64$ (95\% CI: $0.28-0.99)$; flute: $t(36)=$ $3323.73, p=0.001, d=0.61$ (95\% CI: $0.26-0.96)$; trumpet: $t(36)=5.12, p<0.001, d=0.84(95 \%$ 333 CI: 0.46-1.21)]; there were no significant differences among pairs of the three musical 334 instrument timbres $[t(36) \leq 0.92, p \geq 0.37, d \leq 0.15]$. 


\section{(a)}

\section{Musicians and non-musicians}

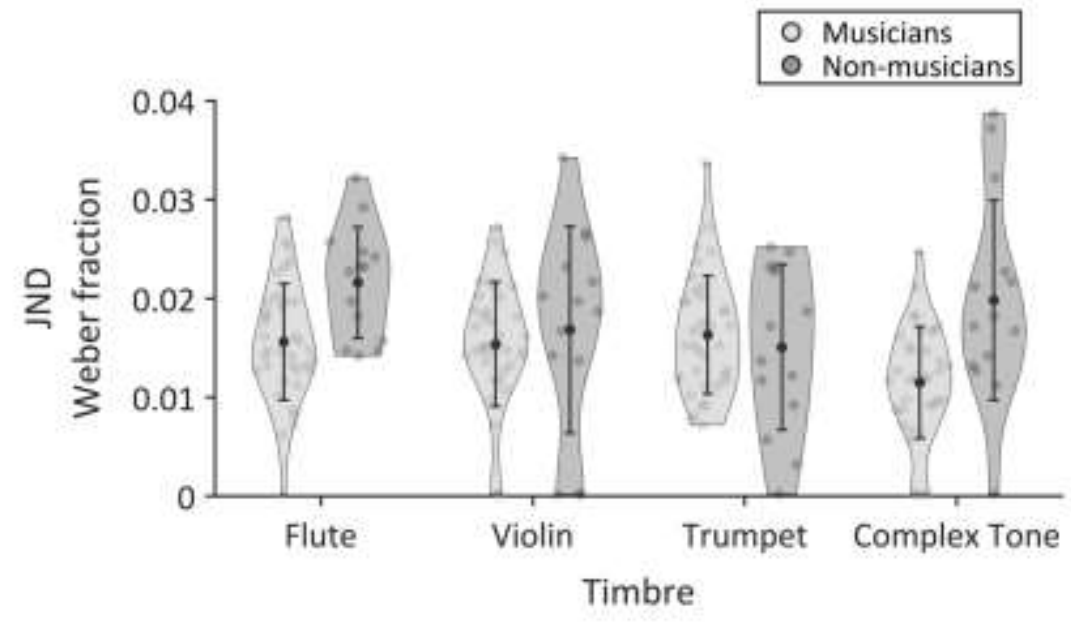

(b) Instrument-specific musical training

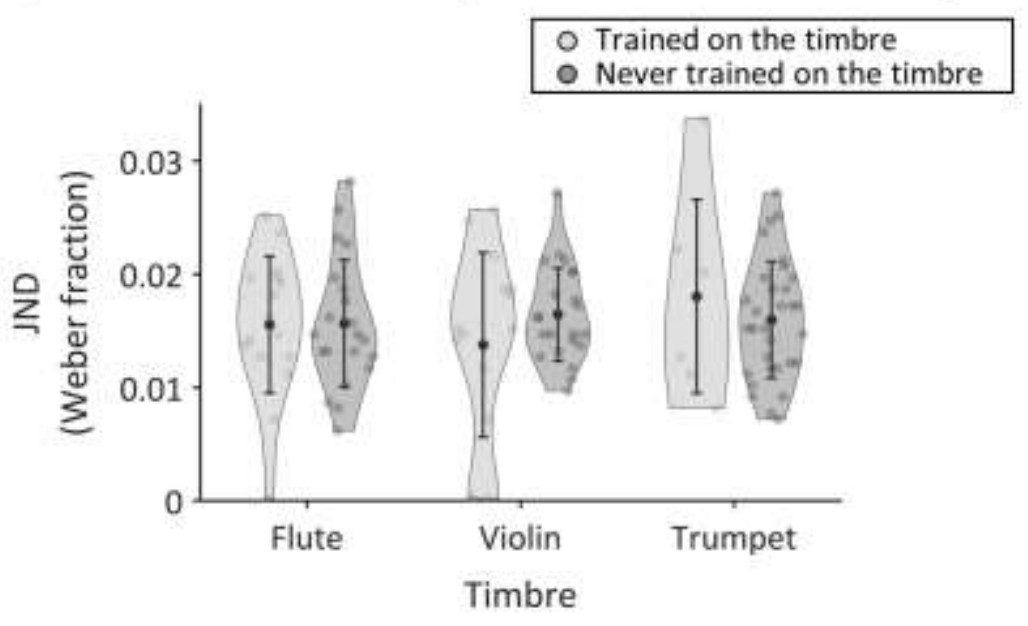

336 Figure 3. Results from the pitch discrimination task, displayed on violin plots. (a) Just noticable

337 difference (JND) for pitch discrimination of sounds of each timbre (flute tones, violin tones,

338 trumpet tones, and flat-spectrum complex tones). Grey dots show results from individual

339 participants, black dots show the mean for each group and timbre, and error bars display one

340 standard deviation of the mean. (b) JND for the three musical timbres (flute tones, violin tones,

341 trumpet tones), separated by participants who were trained and those who were not trained to

342 play the corresponding instrument (e.g., for flute tones, flautists compared with violinists and

343 trumpeters). 


\section{B. Instrument-specific musical training}

Table 2 separates thresholds for each of the Timbres (violin, flute, trumpet, and flat-

347 spectrum harmonic complex tone) by Training group (violinists, flautists, and trumpeters).

349 Table 2. Means for each Training Group across the four Timbres. Brackets indicate 1 standard 350 deviation of the mean.

\section{Timbre}

\begin{tabular}{lcccc}
\hline Training Group & Violin & Flute & Trumpet & Flat-Spectrum \\
& & & & Complex Tone \\
& & & & $0.013(0.006)$ \\
Violinists & $0.014(0.008)$ & $0.014(0.005)$ & $0.014(0.006)$ & $0.011(0.004)$ \\
Flautists & $0.016(0.003)$ & $0.016(0.006)$ & $0.018(0.004)$ & $0.007(0.005)$ \\
Trumpeters & $0.019(0.005)$ & $0.019(0.005)$ & $0.018(0.009)$ & \\
\hline
\end{tabular}

There was a significant main effect of Timbre $\left[F(3,102)=8.68, p<0.001, \omega_{p}{ }^{2}=0.17\right.$

354 (95\% CI: 0.04-0.29)]. Consistent with the analyses reported in the previous section, planned 355 contrasts revealed that listeners had smaller thresholds for flat-spectrum harmonic complex tones 356 than for violin $\left[t(36)=2.83, p=0.008, d_{z}=0.47(95 \%\right.$ CI: $\left.0.12-0.80)\right]$, flute $[t(36)=3.07, p=$ $3570.004, d_{z}=0.50(95 \%$ CI: $\left.0.16-0.84)\right]$, and trumpet $\left[t(36)=3.47, p=0.001, d_{z}=0.57(95 \%\right.$ CI: 358 0.22-0.92)] tones. Thresholds did not differ significantly between the violin, flute, and trumpet 359 tones $\left[t(36) \leq 0.78, p \geq 0.44, d_{z} \leq 0.13\right]$. 
The main effect of Training was not significant $\left[F(2,34)=0.89, p=0.42, \omega_{p}^{2}=-0.01\right.$

361 (95\% CI: 0.00-1.00)], showing that there were no overall differences in discrimination

362 thresholds between participants who were trained on different instruments. However, there was a 363 significant interaction between Timbre and Training $\left[F(6,102)=2.34, p=0.037, \omega_{p}^{2}=0.06\right.$

364 (95\% CI: 0.00-0.12)], showing that thresholds for each timbre differed depending on the 365 instrument that participants were trained to play. However, the interaction was not underpinned 366 by significant differences between Training groups for any of the four Timbres: between-subjects 367 one-way ANOVAs at each Timbre showed no significant effects of Training [violin tones: $F(2$, $36836)=1.40, p=0.26, \omega^{2}=0.02(95 \%$ CI: $0.00-0.15)$; flute tones: $F(2,36)=1.58, p=0.22, \omega^{2}=$ 3690.03 (95\% CI: 0.00-0.18); trumpet tones: $F(2,36)=1.75, p=0.19, \omega^{2}=0.04$ (95\% CI: $0.00-$ 370 0.20); flat-spectrum complex tones: $F(2,36)=3.21, p=0.053, \omega^{2}=-0.11$ (95\% CI: $\left.\left.0.00-0.31\right)\right]$.

371 Instead, the interaction was underpinned by significantly smaller thresholds for the flat-spectrum 372 harmonic complex tone than other timbres among flautists $\left[F(3,45)=5.08, p=0.004, \omega^{2}=0.19\right.$ 373 (95\% CI: $0.00-0.38)]$ and trumpeters $\left[F(3,15)=5.32, p=0.011, \omega^{2}=0.39\right.$ (95\% CI: $0.00-$ $3740.64)]$, whereas the difference was not significant for violinists $\left[F(3,42)=0.08, p=0.97, \omega^{2}=-\right.$ 3750.06 (95\% CI: 0.00-1.00)].

376 Given that there was a trend for violinists to have the most musical training (see Table 1), 377 we investigated whether musical training variables influenced the threshold benefit for the flat378 spectrum complex tone (i.e., difference in JND between the flat-spectrum complex tone and the 379 average of the three musical instrument timbres). The magnitude of the threshold benefit did not 380 correlate with the number of years of musical training $(r=-0.03, p=0.86$; Figure $4 \mathrm{a})$ or the age 381 at which the participant began musical training $(r=0.21, p=0.20$; Figure $4 \mathrm{~b})$. 
Figure $3 b$ separates thresholds for each of the three instruments by whether participants

383 were trained on the timbre or not. Planned independent samples $t$-tests confirmed there was no 384 significant difference in thresholds for violin tones depending on whether or not participants 385 were trained to play the violin $[t(18.79)=1.14, p=0.27, d=0.39$ (95\% CI: $-0.28-1.04)]$, no 386 significant difference in thresholds for flute tones depending on whether or not participants were 387 trained to play the flute $[t(35)=0.05, p=0.96, d=0.02$ (95\% CI: $-0.63-0.67)]$, and no 388 significant difference in thresholds for trumpet tones depending on whether or not participants 389 were trained to play the trumpet $[t(5.62)=0.52, p=0.62, d=0.18$ (95\% CI: $-0.65-1.11)]$.

390 To investigate whether musical training variables influenced the threshold benefit for the 391 trained instrument (i.e., difference in JND between the trained and the average of two untrained 392 instruments), we calculated Spearman's rank correlation coefficients. The magnitude of the 393 threshold benefit did not correlate with the number of years of training on the instrument $(r=$ $3940.04, p=0.80$; Figure $4 c$ ) or the age at which the participant began learning the trained 395 instrument $(r=-0.14, p=0.39$; Figure $4 d)$. There was no difference in the threshold benefit 396 between participants who regularly played their trained instrument (mean $=0.0025$, s.d. $=$ 397 0.0076) and those who were no longer practicing it at the time of the experiment $($ mean $=-$ 3980.0017 , s.d. $=0.0082)[t(35)=1.57, p=0.13, d=0.53(95 \%$ CI: $-0.15-1.19)]$. 
(a)

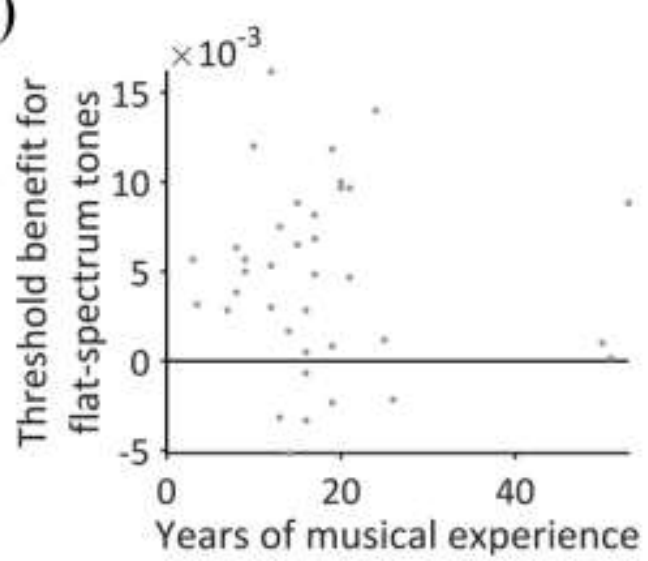

(c)

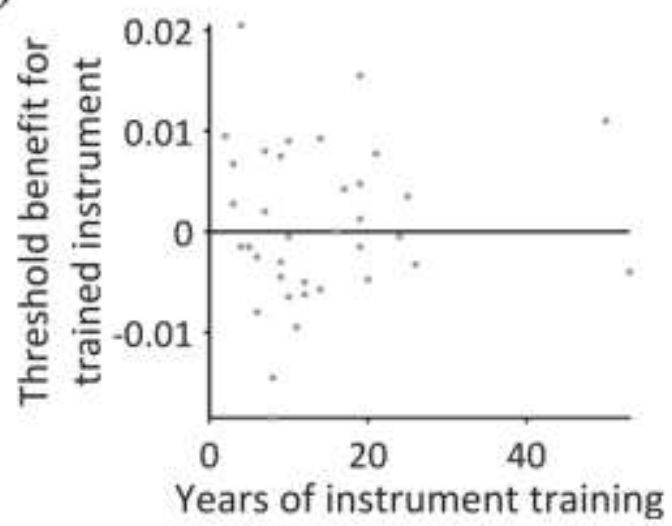

(b)

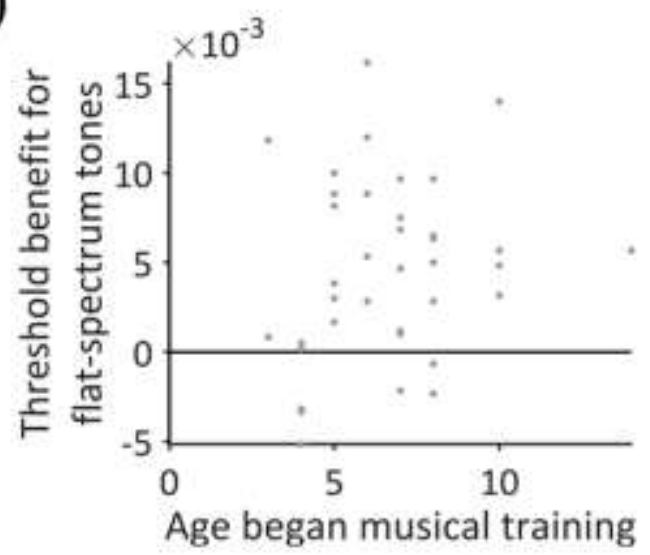

(d)

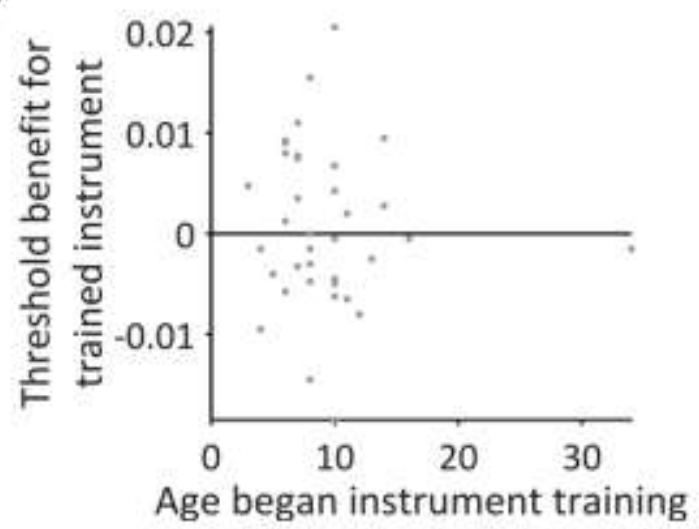

401 Figure 4. Scatter plots showing relationships between training variables and thresholds. The 402 upper row shows relationships between the threshold benefit for flat-spectrum complex tones 403 compared to musical tones and (a) the number of years participants received musical training and 404 (b) the age participants had begun musical training. The lower row shows relationships between 405 the threshold benefit for the trained instrument compared to untrained instruments and (c) the 406 number of years participants had been trained on the instrument and (d) the age participants had 407 started learning the trained instrument. Each dot shows an individual participant (musicians 408 only). 
We found that flautists and trumpeters had better thresholds for discriminating the pitch

412 of flat-spectrum harmonic complex tones than of natural violin, flute, or trumpet tones. The

413 significant interaction we found between Training and Timbre reflects a greater contribution of

414 acoustics to pitch discrimination, than of familiarity with sounds of natural timbres. Our finding

415 implies that flautists and trumpeters can use the additional acoustic information contained in flat-

416 spectrum harmonic complex tones (i.e., greater intensities at the harmonics) to help discriminate

417 pitch. In contrast, a benefit based on familiarity would lead to better thresholds for notes played

418 by musical instruments - because those sounds are heard regularly in everyday life — than for

419 artificial flat-spectrum tones. In addition, we found that thresholds were no better for sounds

420 produced by the instrument that a musician is trained to play than by instruments that they have

421 never been trained to play. This finding suggests that extensive experience with (playing and

422 listening to sounds of) one instrument does not improve pitch discrimination of sounds from that

423 instrument over discrimination of sounds from other musical instruments belonging to different

424 instrument families (i.e., wind, brass, and string).

425 Our finding that pitch-discrimination thresholds do not differ reliably between trained 426 and untrained instruments suggests that memory representations of, and sensorimotor mappings 427 for, pitches of trained instruments do not reliably help listeners to discriminate smaller 428 differences in pitch for timbres of trained instruments than less-familiar timbres. We also found 429 no relationship between discrimination benefits for trained than untrained instruments and either 430 the age that participants started learning the trained instrument or the number of years they 431 played it, suggesting that even extensive experience with sounds of particular timbres does not 432 help discriminate pitch for those timbres over other, untrained, timbres. Possibly, familiarity with 
433 the envelopes or spectra of known timbres and/or enhanced neural representations for sounds

434 played by familiar instruments (Pantev et al., 2001; Shahin et al., 2008; Strait et al., 2012) help

435 musicians to perform other tasks, but our findings imply that they are not used to improve pitch

436 discrimination.

437 Surprisingly, we found that flautists and trumpeters had better discrimination thresholds

438 for artificial complex tones than for musical instrument tones, whereas violinists showed no

439 difference among timbres - and the difference among training groups was confirmed by a

440 significant interaction. Nevertheless, violinists showed a trend in the same direction. There were

441 no significant differences in training demographics among the groups, although there was a trend

442 for the violinists that we recruited to have started training younger and to have continued training

443 for longer than the flautists and trumpeters. Thus, one possible explanation is that earlier and

444 longer musical experience helps to override acoustic advantages for pitch discrimination of flat-

445 spectrum harmonic complex tones. However, we found no significant correlation between the 446 threshold benefit for artificial complex tones (compared with natural instrument tones) and either

447 the age at which participants began musical training or the number of years they received 448 musical training — which is inconsistent with this explanation. Possibly, given that violinists had 449 smaller (albeit not significantly smaller) thresholds for all timbres than flautists and trumpeters, 450 our task may have been less able to detect differences among timbres in violinists. Conversely, 451 this result may reflect true differences in thresholds among musicians who play different 452 instruments, which have been previously reported (e.g., Micheyl et al., 2006), albeit not for the 453 combination of musician groups (violinists, flautists, and trumpeters) studied here. For example, 454 violinists need to tune their instruments and make online adjustments to pitch based on their 455 finger position, whereas flautists and trumpeters do not tune their instruments and make online 
456 adjustments to pitch based on their mouth position. Possibly, this difference could confer a small

457 advantage for discriminating the pitch of natural instrument sounds in violinists, thereby

458 reducing the difference in pitch discrimination between natural and artificial tones.

459 Consistent with the results of previous studies (e.g., Besson et al., 2007; Brown et al., 460 2017; Kishon-Rabin et al., 2001; Magne et al., 2006; Micheyl et al., 2006; Spiegel and Watson, 461 1984), we found better pitch discrimination overall in musicians than non-musicians, suggesting 462 that - in general - musical training improves pitch discrimination. We found that the difference 463 between musicians and non-musicians is most pronounced for artificial flat-spectrum tones and 464 flute tones. A previous study by Micheyl et al. (2006) showed a greater musician benefit for 465 artificial harmonic complex tones than for pure tones. Our results extend those of Micheyl et al. 466 (2006) by showing that the greater musician benefit for artificial complex tones holds when 467 compared to complex tones with less acoustic information, such as natural sounds produced by 468 the violin and trumpet.

469 Our results suggest that musical experience (specifically, training on the flute and 470 trumpet) allows participants to better utilise the additional acoustic information contained in flat471 spectrum harmonic complex tones (i.e., greater intensities at the harmonics) to help discriminate 472 the pitches of these sounds, compared to natural sounds with a skewed distribution of harmonic 473 intensities. Given the results of Moore et al. (2019), this effect is likely due to better auditory 474 sensitivity to pitch cues in musicians, rather than sharper frequency selectivity. However, the 475 mechanism underlying the advantage for flat-spectrum harmonic complex tones is unclear. One 476 possibility is that musicians are better able to use temporal cues conferred by the beating of 477 harmonics within (unresolved) high-frequency channels (Grimault et al., 2002). However, given 478 that these sounds are artificial, this advantage cannot be due to direct experience with sounds, 
479 including experience with high harmonics, which are typically absent in natural music sounds

480 (see Figure 1). Flat-spectrum harmonic complex tones may evoke a more salient pitch than 481 natural instrument sounds, because they contain more harmonic components (Plack and 482 Oxenham, 2006; see Figure 1). However, our finding that discrimination thresholds in musicians 483 are best for flat-spectrum tones is unlikely to be due to salience. If these results were simply due 484 to salience conferred by acoustics, then we should have found better thresholds for flat-spectrum 485 complex tones in non-musicians, and in all of the sub-groups of musicians. Another possibility is 486 that the advantage relates to better working memory for frequency in people with musical 487 experience, which has been previously demonstrated for pure tones (Lad et al., 2020). Our task 488 likely engaged working memory, because it required participants to compare tones that were 489 separated by $1050 \mathrm{~ms}$. Nevertheless, it is unclear why a working memory advantage would be 490 evident for flat-spectrum harmonic complex tones and not for musical instrument tones. Overall, 491 our results suggest that musical training can help listeners to better extract, or hold in memory, 492 pitch based on the acoustic structure of flat-spectrum harmonic complex tones relative to 493 complex tones with a skewed distribution of harmonic intensities-reflecting a musician 494 advantage that has not previously been reported.

495 Broadly speaking, our results add to a wide literature showing that pitch interacts with 496 timbre, demonstrating that these two dimensions are not perceived independently. For example, 497 previous studies have shown that a change in timbre affects pitch comparisons (e.g., Allen and 498 Oxenham, 2014; Krumhansl and Iverson, 1992; Melara and Marks, 1990a, 1990b; Pitt, 1994; 499 Singh and Hirsh, 1992; Vurma et al., 2011; Warrier and Zatorre, 2002). Functional imaging data 500 and research on neurological patients have revealed partially separate neural substrates for pitch 501 and timbre-implicating timbre processing at a higher level-although with some overlap at 
502 early stages of cortical processing (Griffiths et al., 2007; Warren et al., 2005), consistent with

503 behavioural interactions between pitch and timbre. Our study expands upon previous behavioural

504 findings by showing that —even when timbre is held constant — pitch discrimination depends on

505 acoustics of timbre. Specifically, we demonstrated better pitch discrimination for artificial

506 complex tones that contain equal amplitude harmonics than for natural complex tones with a

507 skewed distribution of harmonic intensities, despite greater familiarity with natural sounds.

508 The pitch-discrimination thresholds that we obtained for complex tones in musicians

509 (Weber fraction for 70.0\% JND $=0.015$ ) are higher than those reported for discrimination of

510 complex tones by musicians in three previous studies (Allen \& Oxenham, 2014: Weber fraction

511 for $70.7 \%$ JND of approximately $0.8 \%$, corresponding to a Weber fraction of 0.008 ; Brown et

512 al., 2017: 70.7\% JND of approximately 8 cents, corresponding to a Weber fraction of 0.005 ;

513 Micheyl et al., 2006: Weber fraction for $70.7 \%$ JND $=0.001$ ). However, those previous

514 experiments presented two complex tones sequentially with a brief inter-stimulus interval, rather

515 than embedded within a sequence of tones. Given that Spiegel and Watson (1984) found that

516 musicians had higher pitch-discrimination thresholds when they discriminated between pure

517 tones within 10-tone sequences (Weber fraction $=0.06)$ than between pure tones presented

518 sequentially (Weber fraction $=0.001-0.005)$, higher thresholds for complex tones in the current

519 experiment than in previous experiments presenting two tones sequentially (Brown et al., 2017:

520 Micheyl et al., 2006) could occur because our complex tones were embedded within 4-tone

521 sequences. Another possible explanation for differences in thresholds between experiments is

522 tone duration, which is known to influence discrimination thresholds (see Gockel et al., 2007;

523 Kidd and Watson, 1992). The alternative explanation that thresholds in the current experiment

524 were higher than in previous experiments because the musicians did not have sufficient musical 
525 training is unlikely because Besson et al. (2006) found improvements in pitch-discrimination

526 thresholds after only 6 months of musical training — and all of our musicians had musical training

527 for 3 years or longer.

528 We do not have information about whether our participants were able to use absolute

529 pitch, but we do not expect this to influence the results. For example, Micheyl et al. (2006) found

530 no difference in pitch discrimination for pure and complex tones between musicians who 531 possessed absolute pitch and those who did not.

532 In conclusion, we found that musical experience plays a role in enhancing pitch 533 discrimination abilities - and this is due to a better ability to make use of acoustic information in 534 (artificial) stimuli with flat spectra, rather than an enhancement for timbres most similar to those 535 that have been trained (i.e., natural musical instrument sounds). We predicted that natural 536 familiarity with an instrument would lead to better thresholds for discriminating pitch, but we 537 found no evidence for an advantage. Pitch-discrimination thresholds were no better for sounds 538 with highly familiar timbres (i.e., those of instruments the participants were trained to play) than 539 for sounds with less familiar timbres. In fact, pitch-discrimination thresholds were best for the 540 most unfamiliar type of sound - a flat-spectrum harmonic complex tone-indicating that 541 acoustic information influences pitch-discrimination thresholds more than does timbral 542 familiarity.

\section{ACKNOWLEDGEMENTS}

544 This work was supported by funding from a Music Pilot Study Grant from Western 545 University (supported by the Music Cognition and the Brain Initiative, through the Don Wright 546 Faculty of Music, and Research Western) to E.H., E.K., and L.M. 


\section{AUTHOR CONTRIBUTIONS}

E.H., E.K., and L.M. designed the experiment. E.H., E.K., and E.B. collected the data.

E.H. analysed the data. E.H., E.K., L.M., E.B., T.G., and I.J. wrote the paper.

550

551

552

553

554

555

556

557

558

559

560

561

562

563

564

565

566

567

568

569

\section{REFERENCES}

Allen, E. J., and Oxenham, A. J. (2014). "Symmetric interactions and interference between pitch and timbre," J. Acoust. Soc. Am., 135, 1371-1379. doi:10.1121/1.4863269

Amitay, S., Hawkey, D. J. C., and Moore, D. R. (2005). "Auditory frequency discrimination learning is affected by stimulus variability," Percept. Psychophys., 67, 691-698. doi:10.3758/BF03193525

Ari-Even Roth, D., Amir, O., Alaluf, L., Buchsenspanner, S., and Kishon-Rabin, L. (2003). "The effect of training on frequency discrimination: generalization to untrained frequencies and to the untrained ear,” J. Basic Clin. Physiol. Pharmacol., 14, 137-150. doi:10.1515/JBCPP.2003.14.2.137

Assmann, P. F., and Summerfield, A. Q. (1994). "The contribution of waveform interactions to the perception of concurrent vowels," J. Acoust. Soc. Am., 95, 471-484. Retrieved from http://link.aip.org/link/jasman/v95/i1/p471/s1

Atienza, M., Cantero, J. L., and Dominguez-Marin, E. (2002). "The time course of neural changes underlying auditory perceptual learning," Learn. Mem., 9, 138-150. doi:10.1101/lm.46502

Besson, M., Schön, D., Moreno, S., Santos, A., and Magne, C. (2007). "Influence of musical expertise and musical training on pitch processing in music and language,” Restor. Neurol. Neurosci., 25, 399-410. doi:10.1162/jocn.2010.21585

Brown, C. J., Jeon, E.-K., Driscoll, V., Mussoi, B., Deshpande, S. B., Gfeller, K., and Abbas, P. 
J. (2017). "Effects of long-term musical training on cortical auditory evoked potentials," Ear Hear., 38, e74-e84. doi:10.1097/AUD.0000000000000375

572 Darwin, C. J., Brungart, D. S., and Simpson, B. D. (2003). "Effects of fundamental frequency 573 and vocal-tract length changes on attention to one of two simultaneous talkers," J. Acoust. $574 \quad$ Soc. Am., 114, 2913-2922. doi:10.1121/1.1616924

575 Delhommeau, K., Micheyl, C., Jouvent, R., and Collet, L. (2002). "Transfer of learning across 576 durations and ears in auditory frequency discrimination," Percept. Psychophys., 64, 426436. doi:10.3758/BF03194715

Demany, L. (1985). "Perceptual learning in frequency discrimination,” J. Acoust. Soc. Am., 78, 1118-1120. doi:10.1121/1.393034

Demany, L., and Semal, C. (2002). "Learning to perceive pitch differences," J. Acoust. Soc. Am., 111, 1377-1388. doi:10.1121/1.1445791

Eady, S. J., and Cooper, W. E. (1986). "Speech intonation and focus location in matched statements and questions," J. Acoust. Soc. Am., 80, 402-15. Retrieved from http://www.ncbi.nlm.nih.gov/pubmed/3745672

Faulkner, A. (1985). "Pitch discrimination of harmonic complex signals: residue pitch or multiple component discriminations?,” J. Acoust. Soc. Am., 78, 1993-2004. doi:10.1121/1.392656

Flanagan, J. L., and Saslow, M. G. (1958). "Pitch Discrimination for Synthetic Vowels," J. Acoust. Soc. Am., 30, 435-442. doi:10.1121/1.1909640

Gockel, H. E., Moore, B., Carlyon, R., and Plack, C. (2007). "Effect of duration on the frequency discrimination of individual partials in a complex tone and on the discrimination of fundamental frequency,” J. Acoust. Soc. Am., 121, 373-382. doi:10.1121/1.2382476 
593 Griffiths, T. D., Kumar, S., Warren, J. D., Stewart, L., Stephan, K. E., and Friston, K. J. (2007).

594 "Approaches to the cortical analysis of auditory objects," Hear. Res., 229, 46-53. 595 doi:10.1016/j.heares.2007.01.010

596 Grimault, N., Micheyl, C., Carlyon, R. P., and Collet, L. (2002). "Evidence for two pitch 597 encoding mechanisms using a selective auditory training paradigm,” Percept. Psychophys., $598 \quad \mathbf{6 4}, 189-197$. doi:10.3758/BF03195785

599 Hafke-Dys, H., Preis, A., and Trojan, D. (2016). "Violinists perceptions of and motor reactions 600 to fundamental frequency shifts introduced in auditory feedback," Acta Acustica United 601 with Acustica, 102, 155-158. doi:10.3813/AAA.918932

602 Houtsma, A. J. M., and Smurzynski, J. (1990). "Pitch identification and discrimination for 603 complex tones with many harmonics," J. Acoust. Soc. Am., 87, 304-310. doi:10.1121/1.399297

605 Irvine, D. R., Martin, R. L., Klimkeit, E., and Smith, R. (2000). "Specificity of perceptual 606 learning in a frequency discrimination task,” J. Acoust. Soc. Am., 108, 2964-2968.

607 Kidd, G. R. and Watson, C. S. (1992). "The proportion-of-the-total-duration rule for the 608 discrimination of auditory patterns,” J. Acoust. Soc. Am. 92, 3109-3118.

609 Kishon-Rabin, L., Amir, O., Vexler, Y., and Zaltz, Y. (2001). "Pitch discrimination: are 610 professional musicians better than non-musicians?,” J. Basic Clin. Physiol. Pharmacol., 12, 125-143. doi:10.1515/JBCPP.2001.12.2.125

612 Krumhansl, C. L., and Iverson, P. (1992). "Perceptual Interactions Between Musical Pitch and 613 Timbre,” J. Exp. Psychol. Hum. Percept. Perform., 18, 739-751. doi:10.1037/0096$614 \quad 1523.18 .3 .739$

615 Lad, M., Holmes, E., Chu, A., and Griffiths, T. (2020). "Speech-in-noise detection is related to 
auditory working memory precision for frequency,” Sci. Rep., 10, 13997. doi:10.1101/2020.01.22.915165

618 Magne, C., Schön, D., and Besson, M. (2006). "Musician Children Detect Pitch Violations in 619 Both Music and Language Better than Nonmusician Children: Behavioral and Electrophysiological Approaches," J. Cogn. Neurosci., 18, 199-211. doi:10.1162/jocn.2006.18.2.199

Melara, R. D., and Marks, L. E. (1990a). "Perceptual Primacy of Dimensions: Support for a Model of Dimensional Interaction,” J. Exp. Psychol. Hum. Percept. Perform., 16, 398-414. doi:10.1037/0096-1523.16.2.398

Melara, R. D., and Marks, L. E. (1990b). "Interaction among auditory dimensions: Timbre, pitch, and loudness,” Percept. Psychophys., 48, 169-178. doi:10.3758/BF03207084Menning, H., Roberts, L. E., and Pantev, C. (2000). "Plastic changes in the auditory cortex induced by intensive frequency discrimination training," Neuroreport, 11, 817-822. doi:10.1097/00001756-200003200-00032

Micheyl, C., Delhommeau, K., Perrot, X., and Oxenham, A. J. (2006). "Influence of musical and psychoacoustical training on pitch discrimination," Hear. Res., 219, 36-47. doi:10.1016/j.heares.2006.05.004

Miyazaki, K. (1989). “Absolute Pitch Identification: Effects of Timbre and Pitch Region,” Music Percept., 7, 1-14. doi:10.2307/40285445

Miyazono, H., and Moore, B. C. J. (2013). "Implications for pitch mechanisms of perceptual learning of fundamental frequency discrimination: Effects of spectral region and phase,"

638 Moore, B. C. J., Glasberg, B. R., and Proctor, G. M. (1992). "Accuracy of pitch matching for 
pure tones and for complex tones with overlapping or nonoverlapping harmonics," J. Acoust. Soc. Am., 91, 3443-3450. doi:10.1121/1.402832

641 Moore, B. C. J., Wan, J., Varathanathan, A., Naddell, S., and Baer, T. (2019). "No effect of 642 musical training on frequency selectivity estimated using three methods," Trends Hear., 23, 233121651984198. doi:10.1177/2331216519841980

644 Moore, R. E., Estis, J., Gordon-Hickey, S., and Watts, C. (2008). "Pitch discrimination and pitch 645 matching abilities with vocal and nonvocal stimuli," J. Voice, 22, 399-407. doi:10.1016/j.jvoice.2006.10.013

Pitt, M. A. (1994). "Perception of Pitch and Timbre by Musically Trained and Untrained
Listeners," J. Exp. Psychol. Hum. Percept. Perform., 20, 976-986. doi:10.1037/0096-

Pitt, M. A. (1994). "Perception of Pitch and Timbre by Musically Trained and Untrained
Listeners," J. Exp. Psychol. Hum. Percept. Perform., 20, 976-986. doi:10.1037/0096-

Pantev, C., Roberts, L. E., Schulz, M., Engelien, A., and Ross, B. (2001). "Timbre-specific enhancement of auditory cortical representations in musicians," Neuroreport, 12, 169-174. doi:10.1097/00001756-200101220-00041 1523.20 .5 .976

Plack, C. J., and Oxenham, A. J. (2006). "The Psychophysics of Pitch,” In C. J. Plack, A. J. Oxenham, and R. R. Fay (Eds.), Pitch Neural Coding Percept., Springer-Verlag, New York, 1st ed., pp. 7-55. doi:10.1007/0-387-28958-5_2

Russo, F. A., and Thompson, W. F. (2005). “An interval size illusion: The influence of timbre on the perceived size of melodic intervals," Percept. Psychophys., 67, 559-568. doi:10.3758/BF03193514

Salzberg, R. S. (1980). "The Effects of Visual Stimulus and Instruction on Intonation Accuracy of String Instrumentalists,” Psychol. Music, 8, 42-49. doi:10.1177/030573568082005

Shahin, A. J., Roberts, L. E., Chau, W., Trainor, L. J., and Miller, L. M. (2008). "Music training 
leads to the development of timbre-specific gamma band activity," Neuroimage, 41, 113122. doi:10.1016/j.neuroimage.2008.01.067

664 Singh, P. G., and Hirsh, I. J. (1992). "Influence of spectral locus and F0 changes on the pitch and timbre of complex tones," J. Acoust. Soc. Am., 92, 2650-2661. doi:10.1121/1.404381

Sinnott, J. M., Peterson, M. R., and Hopp, S. L. (1985). "Frequency and intensity discrimination in humans and monkeys,” J. Acoust. Soc. Am., 78, 1977-1985. doi:10.1121/1.392654

Spiegel, M. F., and Watson, C. S. (1984). "Performance on frequency-discrimination tasks by

Strait, D. L., Chan, K., Ashley, R., and Kraus, N. (2012). "Specialization among the specialized: Auditory brainstem function is tuned in to timbre," Cortex, 48, 360-362. doi:10.1016/j.cortex.2011.03.015

Vurma, A., Raju, M., and Kuuda, A. (2011). "Does timbre affect pitch?: Estimations by musician and non-musicians," Psychol. Music,

39, 291-306. doi:10.1177/0305735610373602

679 Warrier, C. M., and Zatorre, R. J. (2002). "Influence of tonal context and timbral variation on

Warren, J. D., Jennings, A. R., and Griffiths, T. D. (2005). “Analysis of the spectral envelope of sounds by the human brain,” Neuroimage, 24, 1052-1057. perception of pitch,” Percept. Psychophys., 64, 198-207. doi:10.3758/BF03195786 doi:10.1016/j.neuroimage.2004.10.031

Wright, B. A., and Fitzgerald, M. B. (2005). "Learning and generalization on five basic auditory discrimination tasks as assessed by threshold changes," Audit. Signal Process., , doi: 10.1007/0-387-27045-0_62.doi:10.1007/0-387-27045-0_62 
685 Interval Discrimination,” (R. Balasubramaniam, Ed.) PLoS One, 8, e75410.

686 doi:10.1371/journal.pone.0075410

687 Zeitlin, L. R. (1964). "Frequency Discrimination of Pure and Complex Tones Published by the 688 Acoustical Society of America,” J. Acoust. Soc. Am., 36, 1027.

689 